\title{
CORRELATION OF STRUCTURAL AND SPECTROSCOPIC PROPERTIES OF A PHOTOSYNTHETIC REACTION CENTER
}

\author{
W. ZINTH, E.W KNAPP, S.F. FISCHER, W. KAISER \\ Physık Departmeni der Technischen Universıat Mwıchen, D-8000 Munich, Federal Republic of Germany'
}

J. DEISENHOFER and H. MICHEL

Max-Planck-Insritur für Biochemie D-g033 Martinsried, Federal Republtc of Germany

Received 10 June 1985

\begin{abstract}
Polarized spectra of absorption and light-induced absorbance changes are presented for the crystallized reaction centers of Rhodopseudomonas viridis We find that a model based on extended dipole interaction between all six pigments is capable of interpreting detailed features such as the contributions from the individual pigments to the various absorption peaks. Even though the pigments are arranged in approximate $C_{2}$ symmetry, the optical spectra together with the calculations rehect deviations from this symmelry, which may be important in understanding the electron pathway
\end{abstract}

\section{Introduction}

In photosynthesis a reaction center catalyses the charge separation and the transfer of an electron across the photosynthetic membrane. The energy for this process is derived from light absorbed by the reaction center itself or by the antenna pigments. Recently the crystallization of the photosynthetic reaction centers from the purple bacterium Rhodopseudomonas viridis [1] allowed the determination of the spatial structure of the reaction center complex by X-ray crystal structure analysis [2] . So far optical and magnetic resonance studies $[3,4]$ have been used to deduce indirect information about the structure and the electron-transfer mechanism. Now theoretical studies based on the structural information can be applied to analyze spectra in more detail and to extract information about the interaction mechanisms and the dynamics of energy and charge transfer.

In fig. 1a the spatial arrangement of the pigment molecules is shown. The phytyl tails of the bacteriochlorophyl b (BClb) and the bacteriopheophytin b (BPhb) molecules as well as the protein components are omitted. The electron density map used to build up the molecular model was determined at a resolu- tion of $3 \mathrm{~A}$ [2]. The estimated precision in the location of the atoms is approximately $0.5-1 \AA$. We summarize a few basic results of the $\mathrm{X}$-ray analysis [2] : Thiere is a local symmetry axus (twofold rotation), the broken line in fig. $1 \mathrm{a}$, with the direction $(x, y, z)=$ $(0.55,0.74,0.40)$ for the tetrapyrrole rings of the $\mathrm{BClb}$ and BPhb. Of particular interest is the arrangement of the two $\mathrm{BClb}\left(\mathrm{BC}_{\mathrm{LP}}, \mathrm{BC}_{\mathrm{MP}}\right)$ which form the special pair (see fig. 1b). Most interestingly in the light of earlier model assumptions $[5,6]$ it is now shown that they overlap most strongly with pyrrole ring I and seem not to be bridged by water molecules. The distance between the planes through the tetrapyrrole systems is $\approx 3 \mathrm{~A}$. The two neighboring $\mathrm{BClb}$ molecules $\left(B_{L A}, B_{M A}\right)$ are very close to the special pair with distances $\left(B C_{L D}, B C_{L A}\right)$ and $\left(B C_{M P}, B C_{M A}\right)$ between the ring centers of $\approx 13 \AA$. Similarly short distances $(\approx 11 \AA)$ are found between the two acces sory BClb $\left(B_{L A}, B C_{M A}\right)$ and their neighboring $B P h b\left(B_{L}\right.$, $\mathrm{BP}_{\mathbf{M}}$ ). Next to the $\mathrm{BP}_{\mathrm{L}}$ molecule is located a menaquinone. A second quinone has not been found so far in the $\mathrm{X}$-ray analysis. 

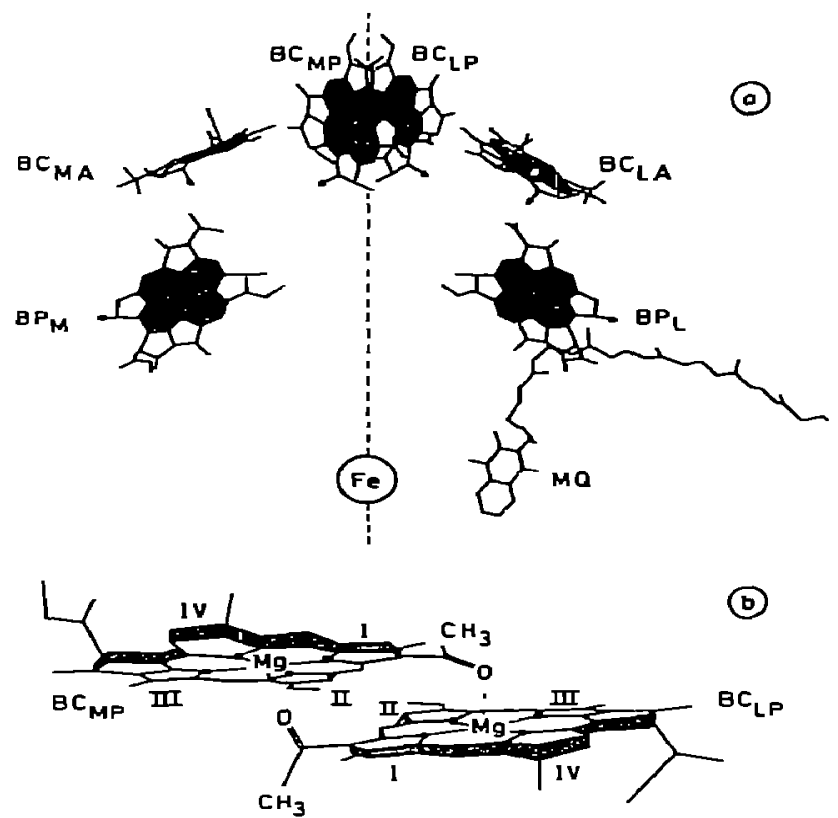

Fig. 1. Molecular arrangement of the pigments of the reaction center: bacteriochlorophyll b (BC), bacteriopheophytin b (BP), the menaquinone (MQ), and the non-heme iron Fe (taken from ref. [2] and modified). The protein part and the heme groups are omitted and the phytyl tals (arrows) are truncated. The indices $L$ and $M$ of the different pigments indicate the proximity to the $L$ and $M$ protein subunits, respectively. The indices $P$ and $A$ denote the special pair and the accessory BClb, respectively. (a) Projection on a plane containing the twofold axis of local symmetry (broken line). (b) Enlarged model of the special pair viewed along the symmetry axis showing details of the binding site of the two BClb molecules important for the prmary charge-separation step A probable orientation of the acetyl group at ring $I$ was chosen.

\section{Results}

The ordered arrangement of the reaction centers in the crystal allows the investigation of well defined polarization geometries not accessible in liquid samples. Fig. 2 shows the absorbance, fig. 3 the absorbance change DA $=A_{\text {exc }}-A_{\text {dark }}$, for different polarization geometries. For the measurement of $A_{\text {exc }}$ the sample was excited with weak continuous actinic light of $\lambda=$ $970 \mathrm{~nm}$ in order to bleach the special pair. The spectroscopic data are taken in the near infrared, where the special pair absorbs and where the $Q_{y}$ bands of

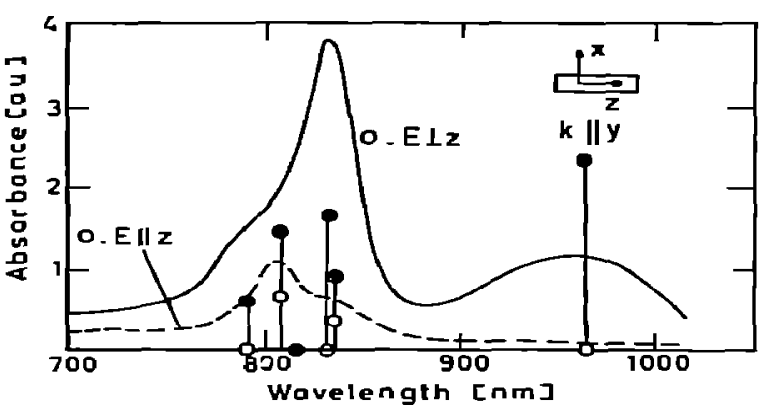

Fig. 2. Absorbance spectra of the crystallized reaction centers for two polarization geometries: beam axis parallel to the crystalline $y$-axis; polarization parallel (broken line) and perpendicular (solid curve) to the tetragonal z-axis. In the crystals the transitions of the BClb and BPhb molecules exhibit a strong dichroism. The transitions are mainly perpendicular to the tetragonal $z$-axis. The vertical bars (with full circles for $E_{\perp z}$ and open circles for $E_{\Downarrow z)}$ indicate the position and strength of the transitions deduced from a theoretical mode! using excitonic coupling of all pigments. The peak around $960 \mathrm{rm}$ is due to the special pair. The absorption around 835 and $795 \mathrm{~nm}$ is mainly due to $\mathrm{BClb}$ and BPhb, respectively.

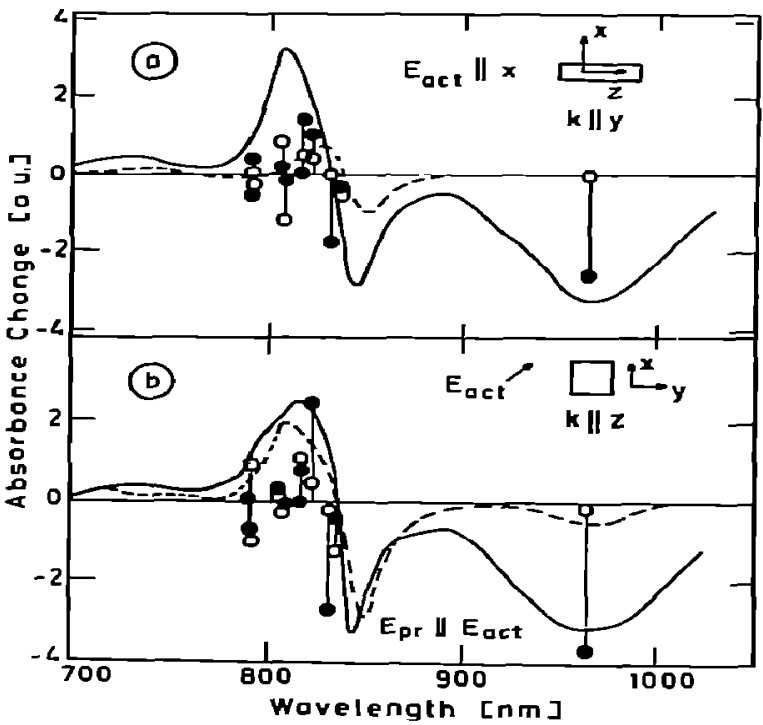

Fig. 3. Change of the absorbance of weak probing light $E_{\mathrm{pr}}$ polarized parallel (solid curve) and perpendicular (broken curve) to the actinic light $E_{\text {rf }}(\lambda \approx 970 \mathrm{~nm})$. (a) Both light beams travel along the crystalline $y$-axis (wavevector $k \| y$ ). The actinic light is polarized perpendicular to the $z$-axis. (b) Both light beams travel along the $z$-axis. Actinic light is polarized at $45^{\circ}$ to the $x$-axis. The absorbance changes calculated from the excitonic coupling model are indicated by vertical bars with full circles ( $\left.E_{\mathrm{pr}} \| E_{\mathrm{act}}\right)$ and open circles $\left(E_{\mathrm{pr}} \perp E_{\mathrm{act}}\right)$. 
the BClb and BPhb are located The experimental apparatus is described in ref. [7].

The close spacing of the six pigments in the reaction center suggests that strong electronic coupling exists between all of them. A model calculation is performed as follows: the Hamiltonian of the coupled system has diagonal elements determined by the frequencies $\epsilon_{n}$ of the uncoupled pigments. The excitonic interaction between couples of pigments $n, m$ (calculated in an extended dipole approximation [4]) determines the coupling (off-diagonal energies $V(n, m))$. The $V(n$, $m$ ) are calculated using the transition dipole moments of the monomers and the atomic coordinates given by the X-ray analysis [2] . The transition frequencies of the excitonically coupled system as well as intensities are obtained from the diagonalisation of the Hamiltonian.

In principle, the calculation could be done without adjusting any parameter. However, the specific solvent shifts of the transition frequencies in the protein environment are not known. The transition dipole moment of the monomers is given experimentally as about $30 \mathrm{D}^{2}$ for bacteriochlorophyll $b$ and $19 \mathrm{D}^{2}$ for bacteriopheophytin $b$ [8]. The transition frequencies of the BClb monomers in solution are at about 12700 $\mathrm{cm}^{-1}$ [9]. In vivo the protein environment is known to red-shift the transition frequency of chlorophyll monomers considerably $[10,11]$. Accordingly, we shifted the BClb transition frequency to $12080 \mathrm{~cm}^{-1}$. Those of the bacteriopheophytins are slightly higher $[9,12]$ in energy $\left(12480 \mathrm{~cm}^{-1}\right)$. A calculation based on these values does not yield the proper splitting of the two pheophytins. In addition, it predicts the location of the low-energy band of the special pair at too high a frequency, at $11450 \mathrm{~cm}^{-1}$ instead of 10370 $\mathrm{cm}^{-1}$. The large shift of the lower special pair band may be due to an enlarged excitonic interaction [13] or a shift of the excitonic state by various interaction mechanisms such as charge transfer, $\mathbf{M g}-\mathrm{O}$ interaction bridging the two monomers (fig. 1b) or interactions with the surrounding protein. Probably several mechanisms contribute. At the moment it is not possible to determine the shift experimentally, since the upper band of the special pair has not been observed. However, prelininary calculations of the circular dichroism spectra based on the same structural information [14] indicate that this band must lie between those of the accessory monomers and the pheophytin bands. With this information the strength of the interaction between $\mathrm{BC}_{\mathrm{LP}}$ and $\mathrm{BC}_{\mathrm{MP}}$ has to be enlarged by a factor of about 1.5, relative to the dipolar interaction, a reasonable enhancement for the closely spaced $\mathrm{BC}_{\mathrm{MP}}-$ $\mathrm{BC}_{\mathrm{LP}} \mathrm{Pair}$ [13]. As a consequerce the frequencies of of $\mathrm{BC}_{\mathrm{MP}}$ and $\mathrm{BC}_{\mathrm{LP}}$ entering the calculation are shifted to $11280 \mathrm{~cm}^{-1}$. The other adjustments of the model concern the transition frequencies of the pheophytins That of $\mathrm{BP}_{\mathrm{M}}$ is raised to $12630 \mathrm{~cm}^{-1}$ while that of $\mathrm{BP}_{\mathrm{L}}$ is lowered to $12330 \mathrm{~cm}^{-1}$. This splitting is likely because of the different protein surroundings. The adjustment of the resonance frequencies of $\mathbf{B P}_{\mathbf{M}}$ and $\mathbf{B P}_{\mathrm{L}}$ is guided by the structural model in combination with the polarized absorption spectrunn. According to this analysis $\mathrm{BP}_{\mathrm{L}}$ should have the lower frequency.

The largest transfer interactions $V(n, m)$ between two pigments at sites $n$ and $m$ are: $V\left(B_{L P}, B C_{M P}\right)=$ $900 \mathrm{~cm}^{-1}, V\left(\mathrm{BC}_{\mathrm{MP}}, \mathrm{BC}_{\mathrm{LA}}\right)=-130 \mathrm{~cm}^{-1} ; V\left(\mathrm{BC}_{\mathrm{LA}}\right.$, $\left.\mathrm{BP}_{\mathrm{L}}\right)=125 \mathrm{~cm}^{-1} ; V\left(\mathrm{BC}_{\mathrm{MA}}, \mathrm{BC}_{\mathrm{LP}}\right)=-115 \mathrm{~cm}^{-1}$; $V\left(\mathrm{BC}_{\mathrm{MA}}, \mathrm{BP}_{\mathrm{M}}\right)=97 \mathrm{~cm}^{-1}$. The othcrs are smaller than $25 \mathrm{~cm}^{-1}$

Table 1

Energies, intensities, polarization, and composition of the exciton states

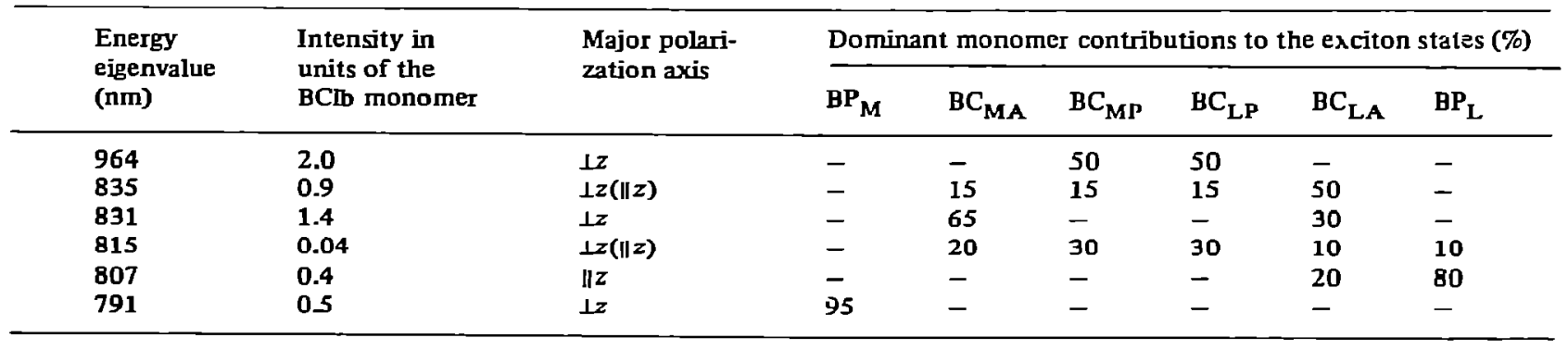


The calculation of the difference spectra (fig. 3) requires a model for the interaction in the illuminated reaction center. Sunce the oxiduzed special pair absorbs in a different frequency regime, it is eliminated from the interaction matrix. In addition, due to the positive charge at the special pair, a shift of the monomers $B C_{M A}$ and $B C_{L A}$ by $200 \mathrm{~cm}^{-1}$ (Stark shift [15]) is introduced.

The results of the calculations are shown by bars in figs 2 and 3 . The wavelengths, intensities, and composition of the bands of the coupled system are presented in table 1 .

The main results can be sumunarized as follows.

(1) The special pair (its lower band) is largely de. coupled from the other pigments. It carries the oscillator strength of two monomers. The upper band predicted at $815 \mathrm{~cm}^{-1}$ rarries almost no intensity (4\% in units of one $\mathrm{BClb}$ monomer). It has a relatively strong admixture from $\mathrm{BC}_{\mathrm{MA}}(17 \%)$ and $\mathrm{BP}_{\mathrm{L}}(11 \%)$.

(2) The bands of the accessory monomers are at 835 and $831 \mathrm{~nm}$. The one at $835 \mathrm{~nm}$ is strongly mixed with all other pigments except $\mathbf{B P}_{\mathbf{M}}$. This explains the existence of the component $z$ at $835 \mathrm{~nm}$ which stems from the coupling to $\mathrm{BP}_{\mathbf{L}}$ (fig. 2). Thus mixing is also reflected in the absorbance changes (figs. $3 \mathrm{a}, 3 \mathrm{~b}$ ).

(3) The decrease of the absorption around $845 \mathrm{~nm}$ is due to the band shift of $\mathbf{B C}_{L_{\mathbf{A}}}$ mixed with $\mathbf{B P}_{\mathbf{L}}$ (figs. 3a, 3b). It is not due to bleaching of the upper special pair transition $[8,15]$.

Summarizing, we have shown that the analysis of $\mathrm{X}$-ray structure and polarized absorption data helps in understanding the intcraction between the pigments in the photosynthetic reaction center.

\section{Acknowledgement}

This work was supported by the Deutsche Forschungsgemeinschaft (SFB 143).

\section{References}

[1] H. Michel, J Mol. Biol. 158 (1982) 567.

[2] J Deisenholer, O. EPp, K. Miki, R. Huber and $H$. Michel, J. Mol. B1ol. 180 (1984) 385.

[3] W.W. Parson, Ann. Rev. Bioeng. 11 (1982) 57.

[4] R.M. Pearlstein, in: Photosynthesis- energy conversion by plants and bacterna, Vol. 1, ed. Govindyec (Academic Press, New York, 1982) pp. 293-330.

[5] J.J. Katz, J.R. Norris and L.L. Shjpman, Brookhaven Symp. Biol 2B (1976) 16.

[6] W. Lubitz, S. Lendzian, H. Scheer, J. Gottstein, M. Plato and K. Mobius, Proc. Natl. Acad. Sci. US 81 (1984) 1401 -

[7] W. Zinth, W. Kaiser and H. Miche], Biochim. Bjophys. Acta 723 (1983) 128 .

[8] V_A. Shuvalow and A_A. Asadov, Biochim. Biophys. Acta 545 (1979) 296.

[9] M.S. Davis, A. Forman, L.K. Hanson, J.P. Thomber and J. Fajer, J. Phys. Chem. 83 (1979) 3325.

[10] R.C. Davis and R.M. Pearlstein, Nature 280 (1979) 413.

[11] J. Eccles and B. Honig, Proc. Natl. Acad Sci. US 80 (1983) 4959.

[12] M. van der Rest and G. Gingras, J. Biol. Chem. 249 (1974) 6446.

[13] A. Warshel, J. Am. Chem. Soc. 101 (1979) 744.

[14] E.W. Knapp, W. Zinth, M. Sander, S.F. Fischer, W Kaiser, J. Deisenhofer and $H$. Michel, to be published.

[15] A. Vermeglio and G. Paillotin, Biochim. Biophys. Acta $681(1982) 32$ 